\title{
Acoustic behaviour of southern right whales in relation to numbers of whales present in Walker Bay, South Africa
}

\section{LH Hofmeyr-Juritz and PB Best}

Mammal Research Institute, University of Pretoria, c/o IzikoSouthAfricanMuseum, PO Box 61, Cape Town 8000, South Africa

Estimating numbers of whales present in an area from recorded call rates could be a useful conservation tool. We recorded southern right whale Eubalaena australis vocalisations and presence in Walker Bay on the south coast of South Africa. In all, 45 sessions with synchronous acoustic and visual data were analysed to determine call rates directly (overall call rate, OCR) and in relation to the number of whales sighted (call rate per whale, CPW) and number of groups sighted (call rate per group, CPG). The OCRs were examined in the presence of varying numbers of whales, using a loglinear model to investigate the dependence of the call rate on whale density. The number of whales present exerted a strong quadratic effect on the OCR, which peaked at around 15 whales and decreased to a low rate as whale presence approached a maximum, for all calls combined and for four of their constituent 13 call types: a quadratic trend was present to varying degrees among the remaining call types. Both quadratic and linear trends were absent when OCR was assessed against number of groups present, possibly because group size increased with increasing density of whales. A linear regression on the CPW and CPG suggested that there was a negative inverse relationship with the number of whales and groups present respectively. These findings are important in that, while they preclude the estimation of absolute numbers from call rates, they imply that under the conditions prevailing in Walker Bay, southern right whales were aware of the presence, arrival and departure of other animals in their vicinity and adjusted their vocal behaviour accordingly.

Keywords: acoustics, call rates, Eubalaena australis, log-linear model, southern right whale, vocalisations, relative abundance

\section{Introduction}

The ability to locate and estimate the density and movement patterns of whales present in an area is an important prerequisite for many aspects of their 
conservation, but such factors are often difficult to ascertain. Whales spend much of their time submerged, and may cover long distances daily. Visual sightings are severely hampered by rough weather conditions, and are almost impossible at night. Communication among individual cetaceans in the contexts of reproductive display, prey detection, predator avoidance and navigation, is primarily acoustic (Clark 1982, MacLennan and Simmonds 1992, Parks 2003a, Parks et al. 2005). Therefore, using biological sounds to detect whales (Mellinger et al. 2011) is suited both to the underwater medium and the properties of the calls. While this method can only detect vocalising individuals, acoustic detection of whales has several advantages over visual detection, as has been demonstrated for fin whales Balaenoptera physalus (McDonald and Fox 1999). It can be applied in remote situations and for extended periods of time (Clark et al. 2010, Mellinger et al. 2011), in situations where boat and aerial surveys would be impractical or expensive. Additionally, remote acoustic detection can cover a greater range than visual detection, can occur under adverse weather conditions and during both day and night, often resulting in greater efficiency (Clark et al. 1996, IFAW 2001).

For the purposes of remote sensing, whale calls are recorded in the absence of manned stations; data are post-processed to count and categorise the calls and to derive absolute rates of sound production. Of cardinal importance is an understanding of the relationship between the absolute call rate and the number of whales present in the area. In some studies, such as for the blue (Balaenoptera musculus) and fin whale (Širović et al. 2004), call production has been used as a 'proxy' for whale presence. Yet, it is possible that the rate of sound production may not be directly correlated with the number of whales present or related in a non-linear manner, depending instead on factors such as the season, location, presence of food, time of day, behaviour (socialising, non-socialising) and sex of the caller. The existence or otherwise of a quantitative link between the recordable calls from groups of whales, and their size and composition, is therefore an important aspect in interpreting remotely recorded whale calls. The possibility of estimating abundance based on calling rates could also provide important information for stock management and industrial planning (Matthews et al. 2001). Even where visual and acoustic surveys are not well correlated, acoustic detection can be a key component in monitoring the recovery of endangered populations (Clark et al. 2010).

In investigating the relationship between the calling behaviour of whales and the numbers present, there are several potential problems. Firstly, the distribution and numbers of non-vocalising whales are not available from acoustic data. Secondly, 
without knowledge of individual differences it is not possible to determine how many individuals contribute to the recorded calls. A combination of in situ observations and passive localisation would provide information of the contexts in which calls are made and the proportions of calling animals (Stafford et al. 1998).

Clark et al. (2007) have shown that right whale calls are peculiarly suited for transmission in shallow water, where for instance a $74 \mathrm{~dB}$ contact call might be detected out to ca $45 \mathrm{~km}$ compared to ca $20 \mathrm{~km}$ in deep water. Empirical determination of range for right whale calls has shown that they can be detected out to at least $80 \mathrm{~km}$ (Marques et al. 2011), and possibly as far as $93 \mathrm{~km}$ (Wade et al. 2006) in shelf waters of the Bering Sea , 50-100 m deep, whereas in the Bay of Fundy, where the whales were in water $120-180 \mathrm{~m}$ deep, the maximum range of tonal and gunshot calls was 28.6 and $33 \mathrm{~km}$ respectively, with averages of 10.3 and $10.1 \mathrm{~km}$ (Laurinolli et al. 2003). Clark (in IFAW 2001) estimated the detection range of right whale calls at pop-up hydrophones in the Great South Channel at water depths of 100 to $200 \mathrm{~m}$ to be $18.5 \mathrm{~km}$, or more realistically $9 \mathrm{~km}$. Even at the latter figure, large portions of most of the bays favoured by right whales on the South African coast would be within the acoustic range of individuals within them.

In this study we combined simultaneous acoustic recordings and visual observations of southern right whales in one of these bays, Walker Bay, to examine the relationship between whale abundance and acoustic output. We used a log-linear model to account for parameters including recording effort and numbers of whales in view.

\section{Material and method}

Between June and November 1999, underwater recordings were taken in Walker Bay (area $225 \mathrm{~km}^{-1}$ ) on the south coast of South Africa (Figure 1a), from a $6 \mathrm{~m}$ semi-rigid inflatable boat during daylight hours when southern right whales were present. During $62 \mathrm{~h}$ of acoustic recordings, concurrent visual observations were taken of whales in the vicinity of the boat. This yielded 45 recording sessions (minimum 12 min, maximum $173 \mathrm{~min}$, mean length $63 \mathrm{~min}$ ) comprising $47 \mathrm{~h}$. Where possible, the number of whales in the various groups and their relative age (adult, juvenile, calf) and behaviour were recorded. For convenience, even if a whale occurred singly it is referred to as a group (of one). A cow-calf pair is referred to as a group of two whales. 


\section{Data collection}

A monorecording system consisted of a hydrophone custom-built by the Institute for Maritime Technology, Simon's Town, South Africa. Due to equipment constraints, it was only possible to calibrate the hydrophone between 2 and $20 \mathrm{kHz}$, while it received from $2 \mathrm{~Hz}$ to at least $20 \mathrm{kHz}$ with a sensitivity of $-146.5 \mathrm{~dB}$ re $1 \mathrm{~V} / \mu \mathrm{Pa}$ at 2 $\mathrm{kHz}$, decreasing to $-169 \mathrm{~dB}$ re $1 \mathrm{~V} / \mu \mathrm{Pa}$ at $20 \mathrm{kHz}$ (specified when the volume of the recording device was set at $50 \%$, and reduced by approximately $7 \mathrm{~dB}$ across the frequency range when the volume was set at $25 \%$; additional gain was set to $20 \mathrm{~dB}$ throughout the testing).

The hydrophone was suspended at a depth of $5 \mathrm{~m}$ from a spar buoy and deployed in a manner so that it drifted away from the boat, depending on the prevailing wind and current. Signals received were recorded on an Aiwa portable DAT recorder (model HS-JS165) with a flat response between $20 \mathrm{~Hz}$ and $20 \mathrm{kHz}$ at $85 \mathrm{~dB}$ (or better), modified to run on a $12 \mathrm{~V}$ alarm battery. The volume was set at $50 \%$, and additional gain was set to $20 \mathrm{~dB}$.

The locations of recording sessions mapped in relation to depth contours, and representative distributions of whales in the bay from aerial surveys, are shown in Figures $1 \mathrm{a}$ and $1 \mathrm{~b}$ respectively.

The goal was to maintain continuous recording sessions for as long as possible and establish whether calls could be allocated to a particular group, and whether calls between different groups could be distinguished. Start- and endpoints for sessions were determined solely by weather conditions (discontinued above Beaufort Scale 3) or safety considerations (drifting too close to the shore) and not by the presence of whales or incidence of calls.

During acoustic recording sessions, a continuous watch was kept from an elevated viewing platform $3 \mathrm{~m}$ above the deck for the arrival, presence and departure of whales. From this vantage point, it was possible to record magnetic bearings from the boat to the whales using a hand-held Autohelm electronic compass. Where possible, groups were tracked as they moved through the study area. Care was taken to record each group only once, but duplicate sightings may have occurred in some instances. On the other hand, not all groups that passed through the area within visual range were necessarily detected. These factors are common to many 
sightings surveys and are considered as relative positive and negative biases respectively. In our analyses, it was assumed that the relative biases were unrelated to the actual number of whales present.

\section{Data analysis}

Comparisons of acoustic and visual range

In situ determination of acoustic range was not attempted. In practice this depends not only on the strength and frequency of the transmitted sound but also on the depth of the caller and receiver, the characteristics of the water column and sea floor between them, and the level and frequency range of ambient noise. Many of these factors were unknown, and although simulations are possible if assumptions are made about some of the parameters, it is unclear how realistic the post-hoc estimates of acoustic range would be.

Determination of visual range to any one sighting in Walker Bay was impractical given the constraints of the experiment, but measurements of radial distances to right whale sightings were made as part of a subsequent project, using the same boat with elevated platform and with some observers in common. Of 43 measurements made (using repeat bearings to the same sighting from the track line and simultaneous GPS locations from a moving boat), $49 \%$ were within $1.2 \mathrm{~km}, 75 \%$ within $2.8 \mathrm{~km}$, and $95 \%$ within $5.2 \mathrm{~km}$ of the boat: maximum values at beyond $6 \mathrm{~km}$ were considered unreliable given the limitations of accurate bearing determination at such distances. A realistic estimate of maximum visual range would then be about $5.2 \mathrm{~km}$. The acoustic range is unlikely to be less than this, especially in the shallow waters of Walker Bay, considering the published estimates of acoustic ranges for southern right whales (see Introduction).

The relative sizes of the visual and acoustic fields can be indirectly assessed from a comparison of the overall call rate per group with that recorded in Argentina. In a coastal environment and at a time of year very comparable to the study in Walker Bay, Clark (in Matthews, 2001) assessed the call rate per group as 5.7 per hour. The average call rate per observed group in Walker Bay was 20.67 per hour. If the species calls at the same rate in both localities, then this would imply that $20.67 / 5.7=$ 3.6 times as many whales were in the acoustic field as were in the visual field. Assuming then that the acoustic field was 3.6.times larger than the visual field and that both subtended similar angles, then if the maximum radial visual range $\left(r_{1}\right)$ was $5.2 \mathrm{~km}$, the equivalent radial acoustic range $\left(r_{2}\right)$ would be 


$$
r_{2}=\sqrt{ }(3.6) r_{1}{ }^{2}
$$

or $9.9 \mathrm{~km}$. This would imply that whales could have been detected about half way across Walker Bay.

Given the apparent mismatch in size between the two fields it is clear that incoming calls would include those from animals both within and outside visual range. However, the study was not designed to investigate the absolute relationship between whale calling rates and numbers present; the basic assumption is that the density of whales within the visual field will be correlated with the overall density of whales in the acoustic field, and therefore that the number of received calls per group is a valid (albeit upwardly biased) indicator of their acoustic activity.

Definition of recording sessions and protocol for defining calls and group size

Only data collected during periods in which there was an overlap of continuous visual observation and acoustic recording were used. At each recording session, the whale count began at zero. Each session was treated as a separate sample; sessions shorter than 12 minutes were excluded or added to an adjacent session, depending on the duration of the inter-session interval.

During post-processing, tapes were monitored aurally using headphones and all calls were scored against time of occurrence. Background noise such as that caused by 'snapping shrimp', mainly related to the proximity of reefs, did not interfere noticeably with call detection. Calls were grouped according to four principal acoustic contours (up, down, flat and variable) and three onset frequencies (Iow $(55-110 \mathrm{~Hz})$, medium $(110-220 \mathrm{~Hz})$ and high $(220-440 \mathrm{~Hz}))$, producing a matrix of 12 call types: low up (LU), medium up (MU), high up (HU); low down (LD), medium down (MD), high down (HD); low flat (LF), medium flat (MF), high flat (HF); low variable (LV), medium variable (MV), and high variable (HV) (Hofmeyr-Juritz 2010). In addition, the whales emitted a non-vocal, broadband, explosive 'gunshot' sound.

Rates of sound production were determined both directly from the number of recorded calls per minute, irrespective of the number of whales sighted from the boat (overall call rate [OCR]), and also in relation to the number of whales sighted (call rate per whale $[\mathrm{CPW}]$ ) or the number of groups seen (call rate per group [CPG]). 
Acoustic recordings and visual observations were made between 23 June 1999 and 20 November 1999.

The accuracy of visually based counts of whales from the viewing platform was dependent on the distance of the whales from the boat as well as prevailing weather conditions. Where possible, the size of groups was specified at each sighting. We used only the final estimate of group size (after the group had been observed for as long as possible). Where it was not possible to estimate group size due to distance, we noted the presence of the group without specifying group size. Subsequently, an averaging algorithm (see below) based on the known sizes of other whale groups present, was used to obtain an estimate of group size.

During a continuous recording session, the number of visible whale groups and their size sometimes changed as whales arrived in or departed from the vicinity of the boat, and joined or left other groups. Groups were tracked through the field of view, and any new group observed during a continuous recording session was added cumulatively to the total number of whales already observed during that recording session; whales already sighted during a continuous recording session were assumed still to be within acoustic range of the hydrophone either before or after they were sighted, and thus able to contribute to the number of recorded calls. Although some new sightings may have been inadvertent re-sightings of the same whale, there were almost certainly other whales within visual range that were never detected.

Rather than ignoring groups of unspecified size, which would clearly have underestimated whale numbers, their size was estimated using a 'clone' algorithm, assuming that the distribution of group sizes was the same for both unspecified and specified groups. Where all groups in a continuous recording session were of unspecified size, the weighted average size of groups in the nearest adjacent recording sessions conducted on the same day were used (with greater weight given to the longer sessions). When the session with unspecified group sizes was either the first or last session of the day, only the subsequent or preceding session was used, respectively. Otherwise, both the preceding and the subsequent sessions were used. In practice, sessions for which group size was calculated from adjacent sessions were separated from the preceding or subsequent session by time intervals ranging from 22 to $72 \mathrm{~min}$. 
There were 45 recording sessions, drawn from 18 research days, in which visual observations were synchronous with acoustic underwater recordings. These were divided into four monthly bins: June (23, 26 and 27), July (20, 21, 26, 28 and 29), September/October (29 and 30 Sep and 1, 2, 4 and 5 Oct), and November (1, 12, 16 and 20). Intervals between sessions (inter-session intervals) on days in which multiple sessions occurred ranged from $7 \mathrm{~min}$ to $3 \mathrm{~h} 52 \mathrm{~min}$ with a mean duration of $58 \mathrm{~min}$. All sessions were treated as independent variables; in each case, the whale and call counts began at zero. During 45 sessions, the total of 430 whales sighted was distributed as follows [\# whales sighted during session (\# sessions)]: [1(1)], [2(1)], [3(4)], [4(4)], [5(4)], [6 (6)], [7(5)], [8(3)], [10(2)], [11(2)], [12(1)], [13(1)], [14(1)], [15(1)], [17(1)], [18(1)], [19(2)], [20(1)], [22(2)], [26(1)], [28(1)].

In total, 3035 calls were used in the analysis.

Statistical analysis

At each session, the number of whales present was recorded. The dependence on this variable of the call rate of a given type per unit time was investigated in two ways. Firstly, for an overview of the nature of the relationship, the CPW was investigated by grouping data in bins of multiples of three whales for aggregation size (that is, 1 to 3 whales, 4 to 6 whales, and so on), representing the number of whales sighted from the boat during any given recording session, and not necessarily all in one group, and calculating the mean CPW for each bin. Because the acoustic activity of a whale may differ depending not only on the number of whales in the bay and the size of the group of which it is a member, but also on the number of groups present, the call rate per group (CPG) was determined in a similar way, by grouping together all instances where the number of groups present was 1 group, 2 groups, and so on, and calculating a mean CPG for each bin. CPW was considered over the whole season and by month, while CPG was considered for the season only. Recordings during which whales were sighted, but apparently did not vocalise, were included in the analyses. Both OCR and CPW were plotted against numbers of whales sighted, and CPG was plotted against numbers of groups sighted, to investigate the relationship between these factors.

For finer resolution of the data, the dependence of the OCR on the number of whales present was analysed with dedicated software (GenStat 12.1), using log-linear models (Mc Cullagh and Nelder 1989) with the following arguments: 
Log [expected number of calls] $=$ constant

+ [linear effect of number of whales]

+ [quadratic effect of number of whales]

Similarly, the model was applied replacing the term 'number of whales' with 'number of groups' (that is, [linear effect of number of groups] and [quadratic effect of number of groups]).

The duration of sessions was taken into account as an offset variable (log [observation period]) which effectively provided a predicted call rate per minute. The models were used on each of the 13 call types and the aggregate number of calls to assess the statistical significance of differing OCRs in the presence of differing numbers of whales and groups. A behavioural factor was not incorporated because of the limited vantage point afforded by the boat to make such observations.

In an earlier analysis of the data, applied both to the 13 call types and to their aggregate, the effects on the OCR due to time of day, month and wind direction and speed had been included, and had generally been found to be non-significant. Consequently the current model made no further reference to these effects, considering instead only the linear and quadratic effects of whale (and group) numbers and recording effort.

\section{Results}

The CPW and CPG, viewed over the whole season, tended to decline consistently in the presence of increasing numbers of whales and groups respectively (Figure 2).

Log-linear model

When investigating the effects of both whale number and group number on the observed variation in call density, random variation was assumed to be consistent with an overdispersed Poisson distribution, on account of the high variability of vocal patterns and calling rates encountered.

\section{LLM: Number of whales}

The predicted number of calls with an increasing number of whales exhibited a marked quadratic effect for certain call types (Figures 3, 4, Table 2). The output of the log-linear model showed that (in decreasing order of strength of effect) MD 
[p=0.004], LV [p=0.012], all calls considered together [ $p=0.023]$, $L U[p=0.027]$, and, to a lesser extent, $L F[p=0.067]$ calls all displayed a strong quadratic effect, in which call numbers increased with an increase in whale numbers but then decreased once some threshold was reached. This effect was not significant for MV $[p=0.102]$ or HD [0.107] although the same trend was evident, and was weak for $M U[p=0.287]$, MF $[p=0.305]$, gunshots [p=0.314], LD [p=0.325], HU [p=0.433] and HF [p=0.684] calls (Table 2).

\section{LLM: Number of groups}

The predicted number of calls measured against an increasing number of groups demonstrated no evidence for a significant quadratic effect, neither for all calls considered together nor for each of the constituent call types. The linear effect was similarly not significant (Table 3), with the exception of MU calls, which approached significance [linear effect for numbers of groups $p=0.053$ ].

When the number of groups sighted for each session was plotted against the number of whales sighted (Figure 5), the relationship between them was best described by a power function $\left(R^{2}=0.6949\right)$, and less convincingly by a logarithmic function $\left(R^{2}=\right.$ $0.5001)$ or a linear function $\left(R^{2}=0.4953\right)$. This indicated that when large numbers of whales were present, group size was likely to be larger as groups amalgamated.

The apparent contrast between the quadratic rise, peak in the presence of around 15 whales and subsequent decline in OCR, and the linear decline in CPW and CPG with aggregation size, is the outcome of an inverse relationship between numbers of whales and CPW per unit time. This may be demonstrated by constructing a simple hypothetical model (Figure 6). Three hypothetical call rates per whale (sparse, medium and abundant) are plotted, each with a maximum CPW when unaccompanied ( $n=1)$, falling linearly in the presence of increasing numbers of whales to a minimum CPW when accompanied by a maximum number of whales (m).

$c^{n}=[(m-n+1) / m] x$

where $c$ is CPW, $n$ is number of whales present, $m$ is maximum number of whales and $x$ is maximum CPW. 
For sparse, medium and abundant calling rates, $c$ is given progressively larger values, producing three calling rates falling linearly with increasing whale numbers.

The OCR is estimated by multiplying the CPW for a given number of whales $(n)$ by that number of whales:

$\operatorname{OCR}=\left(c^{n}\right) n$

The three resultant curves represent the three respective composite call rates with increasing aggregation size (Figure 6).

For a general overview of the OCR, CPW and whale abundance over the whole season, composite data drawn from the results and incorporating all call types were normalised for ease of comparison of trends and plotted for each monthly bin (Figure 7). The inverse relationship between CPW and whale abundance was clearly demonstrated; the OCR continued to rise after the CPW fell in response to an increase in whale numbers (as would be expected) but, as whale numbers continued to rise, the OCR dropped to a minimum, as demonstrated in the simulation (Figure 6) and validated by empirical results of the log-linear model (Figures 3,4 ). This is the natural outcome of the linear drop in the CPW as overall whale numbers increase, as demonstrated in the simulation model (Figure 6) and empirical results (Figures 3, 4). The results, particularly those exemplified in Figure 7 and also in Figures 3 and 4 , clearly demonstrate that, under the conditions of the field experiments, it would not have been possible to estimate absolute whale numbers from call rates in Walker Bay.

\section{Discussion}

The relationship between numbers of groups, numbers of whales and OCR

This relationship emerged as a complex one. (1) Regarding the relationship between number of groups and number of whales, the simplest relationship would have been a linear one (i.e. constant group size). (2) The LLM analysis for numbers of whales present, showing their highly significant quadratic effect on the OCR, would then have been perfectly mirrored in the LLM analysis for numbers of groups present; instead there was no significant evidence for a quadratic effect on the OCR of numbers of groups. (3) The two LLM analyses were based on the same data (the 
same 45 sessions, each of a specific duration, during which a specific number and variety of calls were recorded); the only difference between them was that the unit of measurement of whale density was number of whales present in the one case, and number of groups present in the other.

Further investigation into the relationship between number of whales present and number of groups revealed that it was best described as a power relationship (Figure 5 ), indicating that at larger overall numbers, whales tended to aggregate in larger groups. Such an effect would have tended to reduce the contrast in falling OCR with increasing whale density compared to plotting it against numbers of whales, and most likely accounted for the non-significant results in the LLM 'group' analysis.

\section{Log-linear model}

This study used a log-linear model to investigate the relationship between the overall rate of sound production and the numbers both of whales and of groups present (Mc Cullagh and Nelder 1989). As discussed above, the results for numbers of groups were not significant (Table 3); in contrast the results for numbers of whales were highly significant (Table 2). These results revealed an interesting but confounding aspect of right whale communication: the OCR increased as the number of whales present grew to a certain level, but then dropped off at higher whale densities. This was in contrast to the linear decrease in the CPW as whale numbers increased. Whale behaviour incorporates many contexts, and both the nature and the rate of acoustic communication are linked to specific activities and behavioural states, which are often gender-specific (Clark 1982, 1983,Croll et al. 2002, Parks et al. 2005a). The contexts of the calls in our study are unknown as we could not identify callers in real time. As with other studies using acoustic and visual survey methods (e.g. Clark et al. 2010), the current study was not designed to study behaviour per se, and because of problems associated with a drifting vessel and a low vantage point, it was not possible to identify the caller and place received calls in a behavioural context in real time as calls could be coming from any one or more of groups in the bay, indulging in a variety of behaviours, as would be the case were an autonomous recorder deployed.

Although the quadratic effect is clearer for some call types than others (probably because they are the calls that are most frequently produced, with higher calling 
rates on average), this study provides evidence that there was a tendency for right whales in Walker Bay to vocalize less frequently as whale density increased.

There may be several explanations for this observation.

\section{Eavesdropping}

At high whale densities, silent whales can gather information about the other whales' presence and activities simply by listening. Such 'eavesdropping' has been proposed for rough-toothed dolphins Steno bredansensis, which may benefit from other dolphins' echolocation signals (Götz et al. 2006). It has also been observed that bowhead whales Balaena mysticetus off Point Barrow (at least those within range of the hydrophone) generally appeared to restrict their singing to one whale at a time; when one whale ceased, another often began (Cummings and Holliday 1987). Such behaviour could be particularly beneficial for individuals that might not wish to advertise their presence, such as males competing for access to a receptive female, or females with calves seeking to avoid the attentions of an amorous male.

\section{Focal female effect}

In a fully active surface active group (SAG), the focal whale becomes vocally dominant (Kraus and Hatch 2001, Parks 2003a, b, Parks and Tyack 2005), producing distinct calls that attract males to the SAG (Parks 2003a, b). When the OCR is calculated for such a gathering, especially when there are many males present, as is increasingly the case as the breeding season progresses (Best et al. 2003), the calls of the focal female will make the OCR appear unnaturally low due to the 'dilution' effect of assuming equal input by each whale. In reality, there are times when only one whale is vocally active. However, it could be argued that there might be an increased rate of vocalisation when more competitive males are present. For example, the rate of production of gunshots in the North Atlantic right whale Eubalaena glacialis was weakly correlated with the number of males present in a surface active group (Parks et al. 2005a).

\section{Female harassment avoidance}

Low call rates may reflect a deliberate strategy by females to avoid harassment, whether when alone or accompanying a calf. Southern right whale mothers with calves tend to segregate themselves from other classes, possibly to avoid harassment by males (Elwen and Best 2004 ). Humpback whales Megaptera novaeangliae with calves in Hawaii also chose shallower water than whales without calves, possibly to avoid harassment and injury to calves by sexually active males 
(Smultea 1994). In a visually restricted medium such as the underwater ocean environment, acoustic detection is generally the best option for male suitors. Under such circumstances, there would be a strong selection for the evolution of acoustic detection avoidance strategies for females with or without calves but unwilling to interact with males. The higher the numbers of males present, the stronger the motivation to remain silent, which may be a factor in the marked decline in call density in the presence of high whale numbers in the study area. Right whales with calves move out of nursery areas late in the season, and into regions of the coast such as Walker Bay (Best et al. 2003). Instances of adult interference with cow-calf pairs have frequently been observed in Walker Bay during aerial surveys in October. Many of the whales present off Gansbaai [Figure 1b] in October and November may have been cow-calf pairs, accounting partly for their relative silence. Another possibility is that to avoid detection under such circumstances, acoustic cow-calf interactions would be at a low energy level, and many of their quieter calls would not have been detected by our hydrophones. Clearly, not all whales have similar acoustic behaviour; thus some females may at times be silent to avoid detection while at other times, be vocally dominant in SAGs.

\section{Predator avoidance}

The presence of predators is another potential source of harassment, providing strong motivation for acoustic stealth. Parallel scars, possibly from killer whales, were observed on young calves in Argentinian waters (Thomas and Taber 1984). Not only are calves easy prey for killer whales, but attacks on southern right whale adults have been reported in Peninsula Valdez (Cummings et al. 1972, Thomas and Taber 1984). There are only two reports of such attacks by killer whales in South African waters, and observations are few of southern right whales with rake marks that could be attributable to killer whales (Best et al. 2010). The white shark Carcharodon carcharias is a top predator in Walker Bay and is abundant in the area due to the nearby seal colony at Dyer Island, to the south-east. Opportunistic exploitation of southern right calves by the local great white sharks, whose hearing capabilities, while currently unquantified, are believed to be good below $1000 \mathrm{~Hz}$ (L Compagno, Iziko South African Museum, pers. comm), could cause southern right whales to limit their vocalisation. However, as small gatherings of whales would be subject to the same predation risk as large gatherings, thus being equally motivated to vocalise less, predation avoidance would not drive the observed quadratic net effect observed in Walker Bay, unless larger groupings were more aware of, and more likely quietly to communicate to other whales in the bay, the presence of predators. 


\section{Application of log-linear model results, and the relationship between $O C R$, $C P W$ and whale numbers}

Our results are surprising, and raise many questions about the use of sound by southern right whales; at the same time they appear unequivocally to refute the possibility of using call rates to estimate absolute numbers of whales in Walker Bay, at least using the methodology employed here. The predicted number of calls reached its maximum at a certain threshold and then declined with increasing whale numbers. The largest aggregations of whales were accompanied by a low call rate. Thus southern right whale call rates were not constant, but rather appeared to be inversely related to whale numbers. Under these circumstances, call rates could only be used as conservative estimates of minimum numbers. Minimum population density estimates were calculated in an acoustic survey of fin whale density (McDonald and Fox 1999), using transmission loss models and multipath methods to estimate range from a single hydrophone. Another study using only fixed arrays estimated the population density of North Pacific right whales Eubalaena japonica (Marques et al. 2011). However, where it is possible to monitor the call rate of an individual whale, a change in call rate may indicate a change (possibly inverse) in the group size.

Establishing how many animals are present from recorded calls is a complex problem and many factors may affect call rates, causing variations that confound estimates of animal numbers (Munger et al. 2008,Stafford et al. 2005). A study of Asian elephant Elehas maximus vocalisation patterns by Payne et al. (1998) found that call production rate rose in line with elephant presence, allowing for a relatively simple translation of call density into elephant numbers. However, harp seal Pagophilus groenlandicus vocalisation rates underwater were not related to group size (Serrano and Miller 2000) and there was a poor unreliable relationship between visual sightings and acoustic detection of North Atlantic right whales in Cape Cod Bay (Clark et al. 2010). However, on days when there were both acoustic detections and visual sightings, Clark et al (2010) found a decrease in vocalisations when the number of sightings increased.

The OCR may be estimated without knowledge of whale numbers. However, determining the CPW requires measurement of the OCR and assumes at least knowledge of the number of whales present in the vicinity of where the call originated. Knowledge of the proportion of silent animals and also the identity of the caller for each call would be the ideal situation. Conditions under which this is 
possible are difficult to achieve in studies of non-captive animals, and even more so for cetaceans, with no obvious external sign of vocalisation, so that individual call rates per whale are often approximations rather than precise measurements. An increase in call rate may therefore indicate an individual calling more frequently, or more individuals calling (Munger et al. 2008).

A CPW derived from an OCR is in some respects an artefact. It assumes an equal call rate for each whale present, which is unlikely in reality. The strong quadratic effect observed in LU calls ( $p=0.027$ ) in Walker Bay could be related to their function as contact calls: it is logical that when groupings are large (that is, contact has been established), there would be less motivation to make contact calls, and only a few single (and distant) whales would do so. Thus the estimated CPW would be low, even with fairly frequent calls by one or a few individuals. In Patagonian waters, single southern right whales had higher rates of 'up calls', 'down calls' and 'slaps' than groups containing more than one individual, when mildly active, and production of up calls for single swimming whales was higher than that for those swimming in groups (although high, hybrid and pulsive calls were produced at a higher rate by groups of three rather than two whales (Clark 1983). Nevertheless, individual call rates remain a useful measure provided their interpretation includes the likelihood of different individual call rates within a single group and a dependence on group size, composition and activity. SAG-related calls, or 'screams' (Kraus and Hatch 2001, Parks 2003a), similar to high and hybrid calls (Clark 1983), and the 'medium down' calls described here, which displayed strong quadratic tendencies $(p=0.004)$, are currently believed to be produced by the focal animal in a group of right whales (although the logistics of our study precluded assigning such calls unequivocally to groups exhibiting SAG or any other behaviours).

In this context, the focal animal is responsible for most of the vocalisations, whereas for most of the time the majority of animals are relatively or completely silent. Silent whale groups have been identified in the presence of vocal groups (Clark 1983, Matthews et al. 2001). In the Rottnest Trench, Western Australia, calling blue whales were estimated to represent between 12 and $30 \%$ of the total population in the area (McCauley et al. 2001). Assigning calls of individuals is less problematic when whales are widely spaced over a large area, as blue whales frequently tend to be (Stafford et al. 1998). Right whales are often grouped so closely together that it is impossible to assign calls to an individual. It was conservatively estimated when analysing up call production in Walker Bay, that averaged over the season, at most three-quarters (possible only one-quarter) of southern right whale groups observed 
during recording sessions were vocal, though it was not possible to determine the proportion of silent whales within any group (Hofmeyr-Juritz 2010). Determining the proportion of silent whales involves a calculation both for groups (which is sometimes possible) and for individuals within groups (which presents far greater challenges). Until reliable methods have been developed to establish the separate identities of multiple callers in a group, the estimated rate of sound production for individual whales should be interpreted with caution.

In conclusion, the responsiveness of southern right whales in their calling behaviour to changing numbers of whales present suggests that they were aware of the presence and movements of other whales in Walker Bay. This implies that - based on changing acoustic cues - whales can adjust their vocal behaviour and, importantly, that relative silence is also information. Human-induced marine noise from shipping, leisure and defence activities, as well as oil exploration, is therefore a potential threat to the acoustic environment in which whales live and upon which they depend, and should be monitored closely.

Acknowledgements - This research was supported by the National Research Foundation, the Worldwide Fund for Nature, South Africa (WWF SA), PetroSA (formerly Soekor), the Wildlife and Environment Society, South Africa, and Spescom, to all of whom we are extremely grateful. June Juritz (University of Cape Town) provided invaluable statistical advice. Assistance in the field was primarily provided by Desray Reeb, Rudy Hughes and Vince Calder, while Nigel Coley and Leon Krige assisted greatly with technical and analytical matters. We thank three anonymous reviewers for their helpful comments and suggestions.

\section{References}

Barret-Lennard LG, Ford JKB, Heise KA. 1996. The mixed blessing of echolocation: differences in sonar use by fish-eating and mammal-eating killer whales. Animal Behaviour 51: 553-565.

Best PB. 2000. Coastal distribution, movements and site fidelity of right whales Eubalaena australis off South Africa, 1969-1998. South African Journal of Marine Science 22: 43-55. 
Best PB, Mëyer MA, Lockyer C. 2010. Killer whales in South African waters - a review of their biology. African Journal of Marine Science32: 171-186.

Best PB, Schaeff CM, Reeb D, Palsboll PJ. 2003. Composition and possible function of social groupings of southern right whales in South African waters. Behaviour 140: 1469-1494.

Clark CW. 1982. The acoustic repertoire of the southern right whale: a quantitative analysis. Animal Behaviour 30: 1060-1071.

Clark CW. 1983. Acoustic communication and behaviour of the southern right whale (Eubalaena australis). In: Payne R (ed.), Behavior and communication of whales, American Association for the Advancement of Science Selected Symposia Series 76. Boulder, Colorado: Westview Press. pp 163-198.

Clark CW, Brown MW, Corkeron P. 2010. Visual and acoustic surveys for North Atlantic right whales, Eubalaena glacialis, in Cape Cod Bay, Massachusetts, 20012005: management implications. Marine Mammal Science 26: 837-854.

Clark CW, Charif R, Mitchell S, Colby J. 1996. Distribution and behaviour of the bowhead whale, Balaena mysticetus, based on analysis of acoustic data collected during the 1993 spring migration off Point Barrow, Alaska. Report of the International Whaling Commision 46: 541-552.

Clark CW, Gillespie D, Nowacek DP, Susan E. Parks SE. 2007. Listening to Their World: Acoustics for Monitoring and Protecting Right Whales in an Urbanized Ocean. In (Kraus S \& Rolland R, eds) The Urban Whale. North Atlantic right whales at the crossroads. Harvard University Press.

Croll DA, Clark CW, Acevedo A, Tershy B, Flores T, Gedamke J, Urban J. 2002. Only male fin whales sing loud songs. Nature 417 (6891): 809.

Cummings WC, Holliday DV. 1987. Sound and source levels from bowhead whales off Pt. Barrow, Alaska. journal of the Acoustical Society of America 82 (3): 814-821.

Cummings WC, Fish JF, Thompson PO. 1972. Sound production and other behaviour of southern right whales, Eubalaena australis. Transactions of the San Diego Society of Natural History 17: 1-14. 
Elwen S, Best PB. 2004 Environmental factors influencing the distribution of southern right whales (Eubalaena australis) on the south coast of South Africa II: within bay distribution. Marine Mammal Science 20: 583-601.

Götz T, Verfuß UK, Schnitzler H. 2006. 'Eavesdropping' in wild rough-toothed dolphins (Steno bredanensis)? Biological Letters of the Royal Society 2: 5-7.

Hofmeyr-Juritz L. 2010. The nature and rate of vocalisation by southern right whales (Eubalaena australis), and the evidence for individually distinctive calls. $\mathrm{PhD}$ thesis. University of Pretoria, South Africa.

IFAW (International Fund for Animal Welfare). 2001. Report of the Workshop on Right Whale Acoustics: Practical Applications in Conservation. Yarmouth Port, MA 02675, USA.

Kraus SD, Hatch JJ. 2001. Mating strategies in the North Atlantic right whale (Eubalaena glacialis). Journal of Cetacean Research and Management (Special Issue 2): 237-244.

Laurinolli MH, Hay AE, Desharnais F, Taggart CT. 2003. Localization of North Atlantic right whale sounds in the Bay of Fundy using a sonobuoy array. Marine Mammal Science 19:708-723.

MacLennan DN, Simmonds EJ. 1992. Biological acoustics. In: MacLennan DN, Simmonds EJ (eds), Fisheries acoustics. London, Tokyo, Melbourne, Madras: Chapman \& Hall. pp 89-118.

Marques TA, Munger L, Thomas L, Wiggins S, Hildebrand JA. 2011. Estimating North Pacific right whale Eubalaena japonica density using passive acoustic cue counting. Endangered Species Research 13: 163-172.

Matthews JN, Brown S, Gillespie D, Johnson M, McLanaghan R, Moscrop A, Nowacek D, Leaper R, Lewis T, Tyack P. 2001. Vocalisation rates of the North Atlantic right whale (Eubalaena glacialis). Journal of Cetacean Research and Management 3: 271-282. 
Mc Cullagh P, Nelder J. 1989. Generalized linear models ( $2^{\text {nd }}$ edn). London, New York: Chapman \& Hall. pp 193-244.

McCauley RD, Jenner C, Bannister JL, Burton CLK, Cato DH, Duncan A. 2001. Blue whale calling in the Rottnest Trench - 2000, Western Australia. Curtin University of Technology: Centre for Marine Science and Technology ReportR2001-6: 1-56.

McDonald MA, Fox CG. 1999. Passive acoustic methods applied to fin whale population density estimates. Journal of the Acoustical Society of America 105: 2643-2651.

Mellinger DK, Nieukirk SL, Klinck K, Klinck H, Dziak RP, Clapham PJ, Brandsdóttir B. 2011. Confirmation of right whales near a nineteenth-century whaling ground east of southern Greenland. Biology Letters 7: 411-413.

Munger LM, Wiggins SM, Moore SE, Hildebrand JA. 2008. North Pacific right whale (Eubalaena japonica) seasonal and diel calling patterns from long-term acoustic recordings in the southeastern Bering Sea, 2000-2006. Marine Mammal Science 24: 795-814.

Parks SE. 2003b. Acoustic communication in the North Atlantic right whale (Eubalaena glacialis). PhD thesis, Massachusetts Institute of Technology \& Woods Hole Oceanographic Institute.

Parks SE. 2003a. Response of North Atlantic right whales (Eubalaena glacialis) to playback of calls recorded from surface active groups in both the North and South Atlantic. Marine Mammal Science 19: 563-580.

Parks SE, Tyack PL. 2005. Sound production by North Atlantic right whales (Eubalaena glacialis) in surface active groups. Journal of the Acoustical Society of America 117: 3297-3306.

Parks SE, Hamilton PK, Kraus SD, Tyack PL. 2005. The Gunshot sound produced by male North Atlantic right whales (Eubalaena glacialis) and its potential function in reproductive advertisement. Marine Mammal Science 21: 458-475.

Payne K, Langbauer WR, Thomas E. 1998. Infrasonic calls of the Asian elephant (Elephas maximus). Behavioural Ecology and Sociobiology 18: 297-301. 
Serrano A, Miller EH. 2000. How vocal are harp seals (Pagophilus groenlandicus)? A captive study of seasonal and diel patterns. Aquatic Mammals 26: 253-259.

Širović A, Hildebrand JA, Wiggins SM, McDonald MA, Moore SE, Thiele D. 2004. Seasonality of blue and fin whale calls and the influence of sea ice in the Western Antarctic Peninsula. Deep-Sea Research Part II: Topical Studies in Oceanography 51: 2327-2344.

Smultea MA. 1994. Segregation by humpback whale (Megaptera novaeangliae) cows with a calf in coastal habitat near the island of Hawaii. Canadian Journal of Zoology 72: 805-811.

Stafford KM, Fox CG, Clark DS. 1998. Long-range acoustic detection and localization of blue whale calls in the northeast Pacific Ocean. Journal of the Acoustical Society of America 104: 3616-3625.

Stafford KM, Moore SE, Fox CG. 2005. Diel variation in blue whale calls recorded in the eastern tropical Pacific. Animal Behaviour 69: 951-958.

Thomas PO, Taber SM. 1984. Mother-infant interaction and behavioural development in southern right whales, Eubalaena australis. Behaviour 88: 42-60.

Wade P, Heide-Jørgensen MP, Shelden K, Barlow J, Carretta J, Durban J, LeDuc R, Munger L, Rankin S, Sauter A, and Stinchcomb C 2006. Acoustic detection and satellite-tracking leads to discovery of rare concentration of endangered North Pacific right whales. Biology Letters 2(3): 417-419 
Table 1: Seasonal and monthly percentages of groups of southern right whales of known size, Walker Bay, South Africa

\begin{tabular}{|c|c|c|c|c|c|c|c|c|c|c|}
\hline \multirow[b]{2}{*}{ Period } & \multicolumn{10}{|c|}{ Group size } \\
\hline & $\mathrm{n}$ & 1 & 2 & 3 & 4 & 5 & 6 & 7 & 8 & total \% \\
\hline Season & 98 & 25.5 & 55.1 & 9.2 & 4.1 & 3.1 & 0.0 & 2.0 & 1.0 & 100 \\
\hline June & 10 & 30.0 & 30.0 & 20.0 & 10.0 & 10.0 & 0.0 & 0.0 & 0.0 & 100 \\
\hline July & 26 & 26.9 & 53.8 & 7.7 & 7.7 & 0.0 & 0.0 & 3.8 & 0.0 & 100 \\
\hline Sept/Oct & 28 & 28.6 & 53.6 & 3.6 & 3.6 & 3.6 & 0.0 & 3.6 & 3.6 & 100 \\
\hline Nov & 34 & 20.6 & 64.7 & 11.8 & 0.0 & 2.9 & 0.0 & 0.0 & 0.0 & 100 \\
\hline
\end{tabular}


Table 2: Estimates of parameters from the LLM for all 13 distinct call types of southern right whales in Walker Bay, South Africa, and their aggregate, Allcalls, using a model which takes account of session lengths and the linear and quadratic effects of the number of whales on number of calls. Call parameters are presented in descending order of statistical significance (t pr.) of the quadratic effect of whale numbers on call rates

\begin{tabular}{|c|c|c|c|c|c|c|}
\hline Call type & Parameter & estimate & s.e. & $t(42)$ & t pr. & estimate \\
\hline \multirow[t]{3}{*}{ MD } & Constant & -4.173 & 0.608 & -6.87 & $<.001$ & 0.01541 \\
\hline & Whales linear & 0.3282 & 0.0916 & 3.58 & $<.001$ & 1.388 \\
\hline & Whales quad & -0.0096 & 0.00315 & -3.05 & 0.004 & 0.9904 \\
\hline \multirow[t]{3}{*}{ LV } & Constant & -7.65 & 1.09 & -7.02 & $<.001$ & 0.0004765 \\
\hline & Whales linear & 0.49 & 0.163 & 3.01 & 0.004 & 1.632 \\
\hline & Whales quad & -0.01506 & 0.00571 & -2.64 & 0.012 & 0.9851 \\
\hline \multirow[t]{3}{*}{ Allcalls } & Constant & -1.071 & 0.436 & -2.45 & 0.018 & 0.3426 \\
\hline & Whales linear & 0.1913 & 0.0712 & 2.68 & 0.01 & 1.211 \\
\hline & Whales quad & -0.00597 & 0.00253 & -2.35 & 0.023 & 0.9941 \\
\hline \multirow[t]{3}{*}{ LU } & Constant & -2.682 & 0.628 & -4.27 & $<.001$ & 0.0684 \\
\hline & Whales linear & 0.263 & 0.109 & 2.41 & 0.02 & 1.301 \\
\hline & Whales quad & -0.0096 & 0.0042 & -2.29 & 0.027 & 0.9904 \\
\hline \multirow[t]{3}{*}{$\mathrm{LF}$} & Constant & -4.504 & 0.811 & -5.55 & $<.001$ & 0.01107 \\
\hline & Whales linear & 0.278 & 0.133 & 2.08 & 0.043 & 1.32 \\
\hline & Whales quad & -0.00921 & 0.0049 & -1.88 & 0.067 & 0.9908 \\
\hline \multirow[t]{3}{*}{$\mathrm{MV}$} & Constant & -4.412 & 0.733 & -6.02 & $<.001$ & 0.01213 \\
\hline & Whales linear & 0.225 & 0.12 & 1.88 & 0.067 & 1.252 \\
\hline & Whales quad & -0.00718 & 0.0043 & -1.67 & 0.102 & 0.9928 \\
\hline \multirow[t]{3}{*}{ HD } & Constant & -3.203 & 0.74 & -4.33 & $<.001$ & 0.04066 \\
\hline & Whales linear & 0.221 & 0.128 & 1.73 & 0.091 & 1.247 \\
\hline & Whales quad & -0.00794 & 0.00483 & -1.65 & 0.107 & 0.9921 \\
\hline \multirow[t]{3}{*}{ HV } & Constant & -4.23 & 0.773 & -5.47 & $<.001$ & 0.01456 \\
\hline & Whales linear & 0.203 & 0.123 & 1.65 & 0.106 & 1.225 \\
\hline & Whales quad & -0.00601 & 0.0043 & -1.4 & 0.17 & 0.994 \\
\hline MU & Constant & -3.271 & 0.577 & -5.67 & $<.001$ & 0.03795 \\
\hline
\end{tabular}




\begin{tabular}{|c|c|c|c|c|c|c|}
\hline & Whales linear & 0.1356 & 0.0908 & 1.49 & 0.143 & 1.145 \\
\hline & Whales quad & -0.0033 & 0.00305 & -1.08 & 0.287 & 0.9967 \\
\hline \multirow[t]{3}{*}{ MF } & Constant & -3.272 & 0.567 & -5.77 & $<.001$ & 0.03795 \\
\hline & Whales linear & 0.1352 & 0.0879 & 1.54 & 0.131 & 1.145 \\
\hline & Whales quad & -0.00302 & 0.00291 & -1.04 & 0.305 & 0.997 \\
\hline \multirow[t]{3}{*}{ Gunshot } & Constant & -3.699 & 0.785 & -4.71 & $<.001$ & 0.02475 \\
\hline & Whales linear & 0.152 & 0.129 & 1.18 & 0.245 & 1.164 \\
\hline & Whales quad & -0.00466 & 0.00457 & -1.02 & 0.314 & 0.9954 \\
\hline \multirow[t]{3}{*}{ LD } & Constant & -5.479 & 0.802 & -6.83 & $<.001$ & 0.004175 \\
\hline & Whales linear & 0.158 & 0.129 & 1.23 & 0.226 & 1.171 \\
\hline & Whales quad & -0.00442 & 0.00444 & -1 & 0.325 & 0.9956 \\
\hline \multirow[t]{3}{*}{$\mathrm{HU}$} & Constant & -3.883 & 0.704 & -5.52 & $<.001$ & 0.02059 \\
\hline & Whales linear & 0.104 & 0.12 & 0.87 & 0.391 & 1.11 \\
\hline & Whales quad & -0.00341 & 0.00431 & -0.79 & 0.433 & 0.9966 \\
\hline \multirow[t]{3}{*}{$\mathrm{HF}$} & Constant & -3.662 & 0.598 & -6.13 & $<.001$ & 0.02568 \\
\hline & Whales linear & 0.0625 & 0.0992 & 0.63 & 0.532 & 1.065 \\
\hline & Whales quad & -0.0014 & 0.00341 & -0.41 & 0.684 & 0.9986 \\
\hline
\end{tabular}


Table 3. Ranked probability of linear and quadratic effects of the number of groups present on the predicted OCR, for all 13 distinct call types of southern right whales in Walker Bay, South Africa, and their aggregate, Allcalls.

\begin{tabular}{ccc}
\multicolumn{3}{c}{ ranked linear effect } \\
call type & (linear) $p$ & \% of calls \\
MU & 0.053 & 9.6 \\
MD & 0.093 & 12.6 \\
LU & 0.13 & 25.4 \\
All calls & 0.165 & 100 \\
MV & 0.236 & 4.3 \\
MF & 0.476 & 10.1 \\
HV & 0.488 & 5 \\
LV & 0.52 & 1.1 \\
HF & 0.565 & 3.9 \\
HD & 0.58 & 12.2 \\
HU & 0.616 & 3.5 \\
LD & 0.694 & 1.1 \\
Gunshot & 0.856 & 5.9 \\
LF & 0.91 & 5.2
\end{tabular}

\begin{tabular}{ccc}
\multicolumn{2}{c}{ ranked quadratic effect } & (groups) \\
call type & (quad) $p$ & $\%$ of calls \\
MU & 0.107 & 9.6 \\
LU & 0.17 & 25.4 \\
MD & 0.3 & 12.6 \\
All calls & 0.37 & 100 \\
LD & 0.385 & 1.1 \\
MV & 0.453 & 4.3 \\
LF & 0.618 & 5.2 \\
HF & 0.642 & 3.9 \\
HV & 0.724 & 5 \\
HU & 0.726 & 3.5 \\
HD & 0.783 & 12.2 \\
LV & 0.871 & 1.1 \\
MF & 0.927 & 10.1 \\
Gunshot & 0.963 & 5.9
\end{tabular}



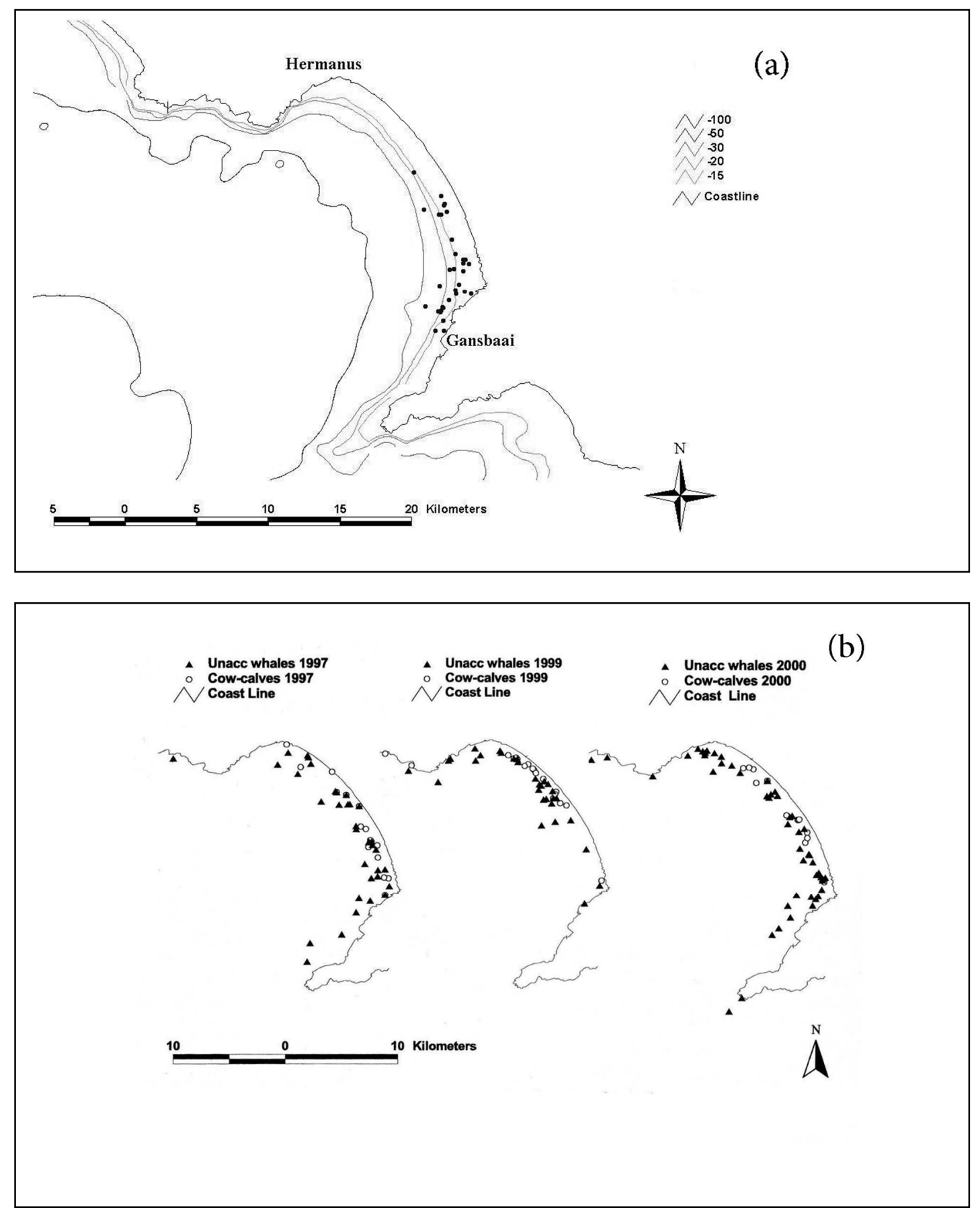

Figure 1 (a).Locations of recording sessions for right whales in Walker Bay, South Africa, in relation to bathymetry. (b).Distribution of right whales seen on October aerial surveys in Walker Bay, 1997, 1999 and 2000 (from (Elwen and Best 2004). 

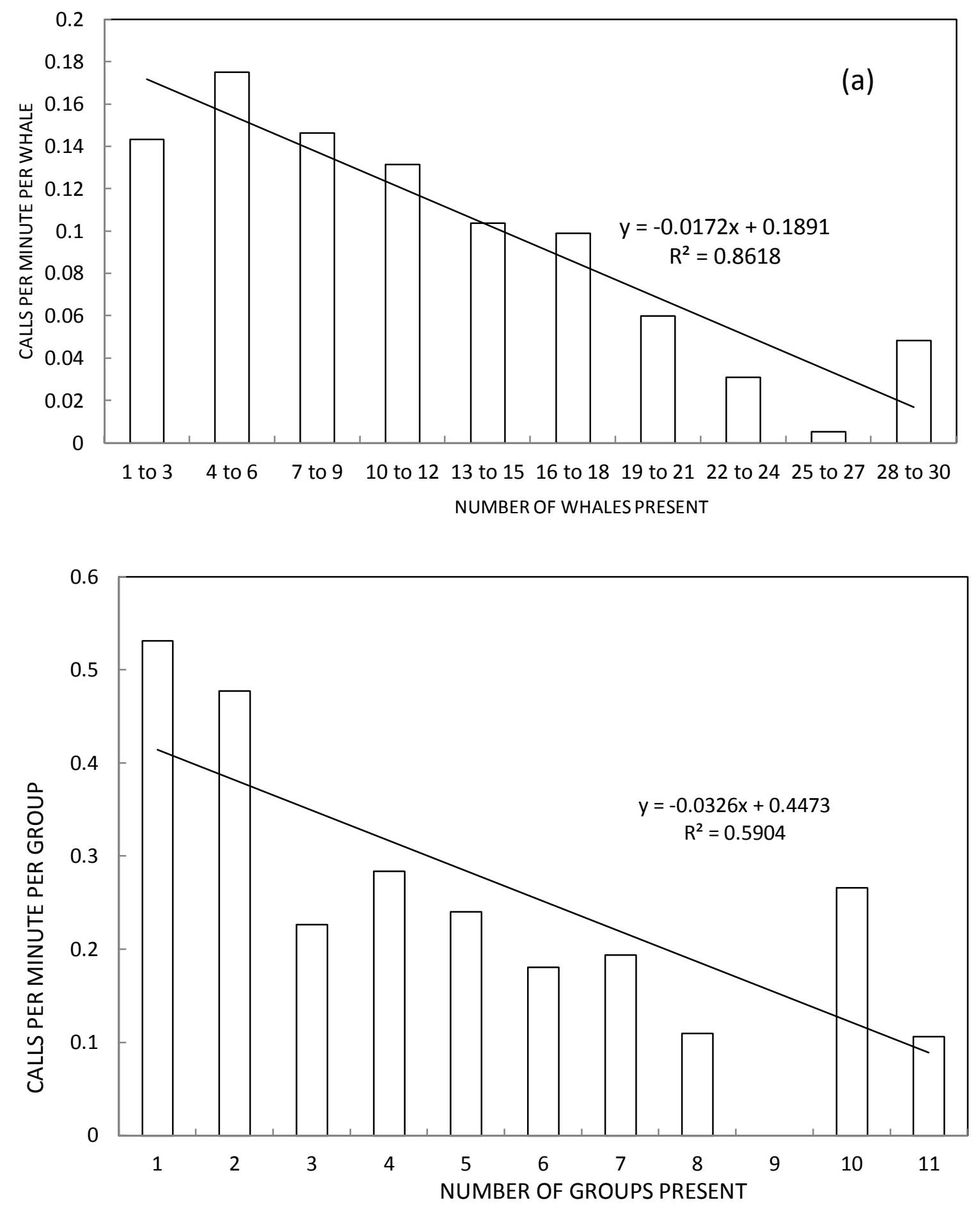

Figure 2. Relationship between (a) call rate per whale (CPW) and numbers of whales present (bin size 3 whales), and (b) call rate per group (CPG) and numbers of groups present (bin size increment 1 group), for all calls of southern right whales over the whole season in Walker Bay, South Africa. 


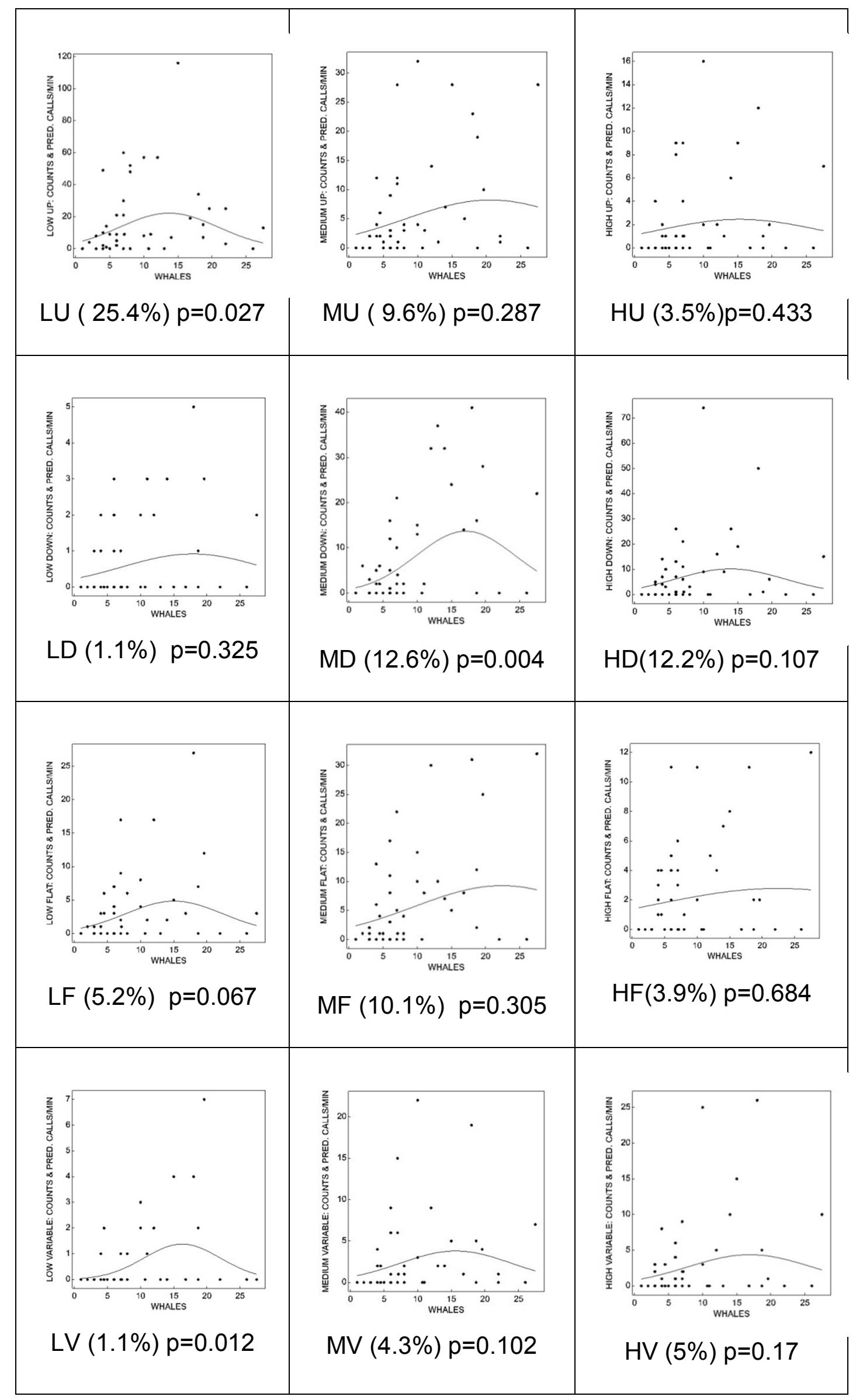


Figure 3. Quadratic effects of numbers of whales on the call rates of 12 vocalised call types of southern right whales.

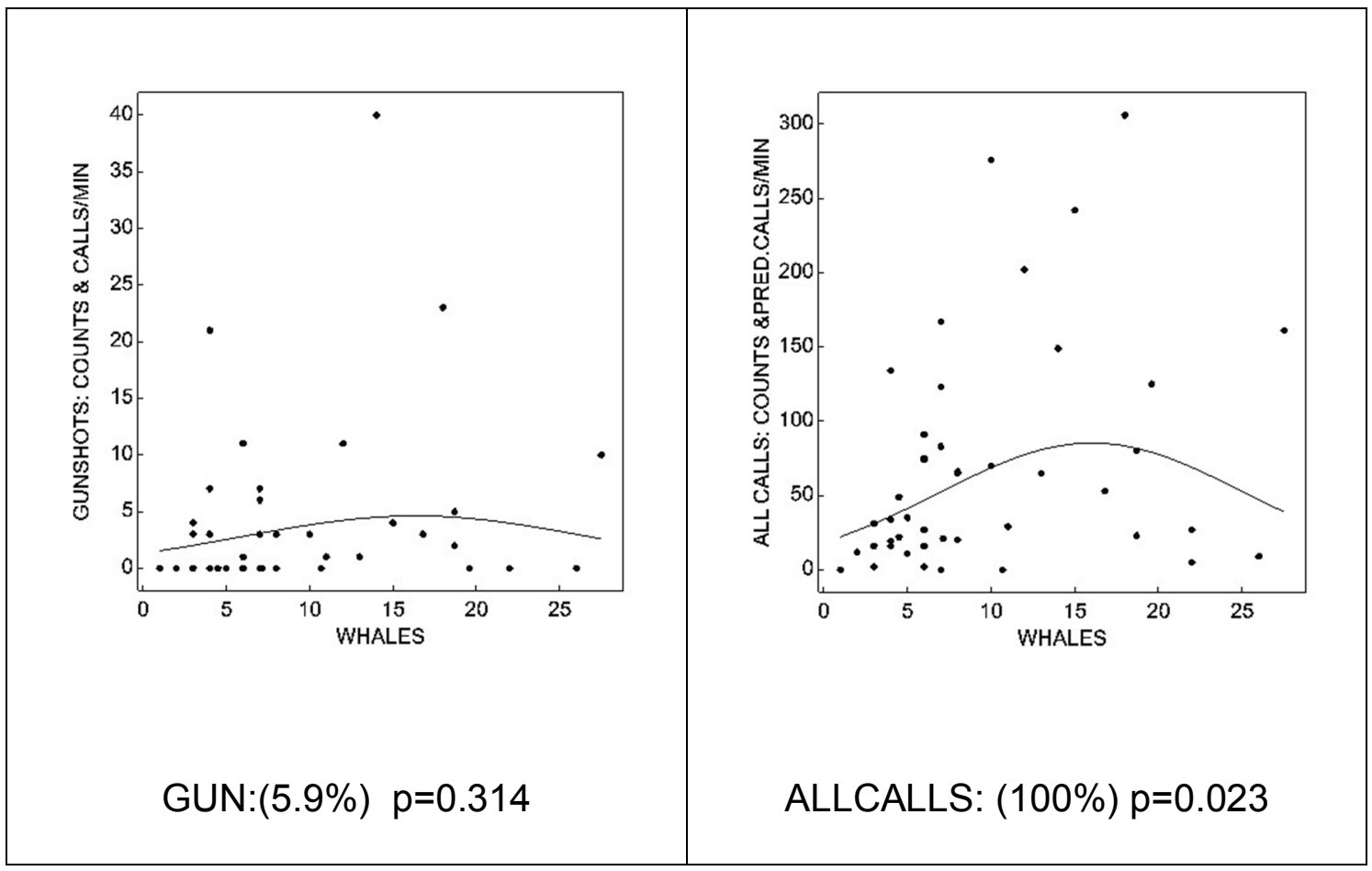

Figure 4. Quadratic effects of numbers of whales on broadband gunshot and ALLCALLS call rates of southern right whales. 


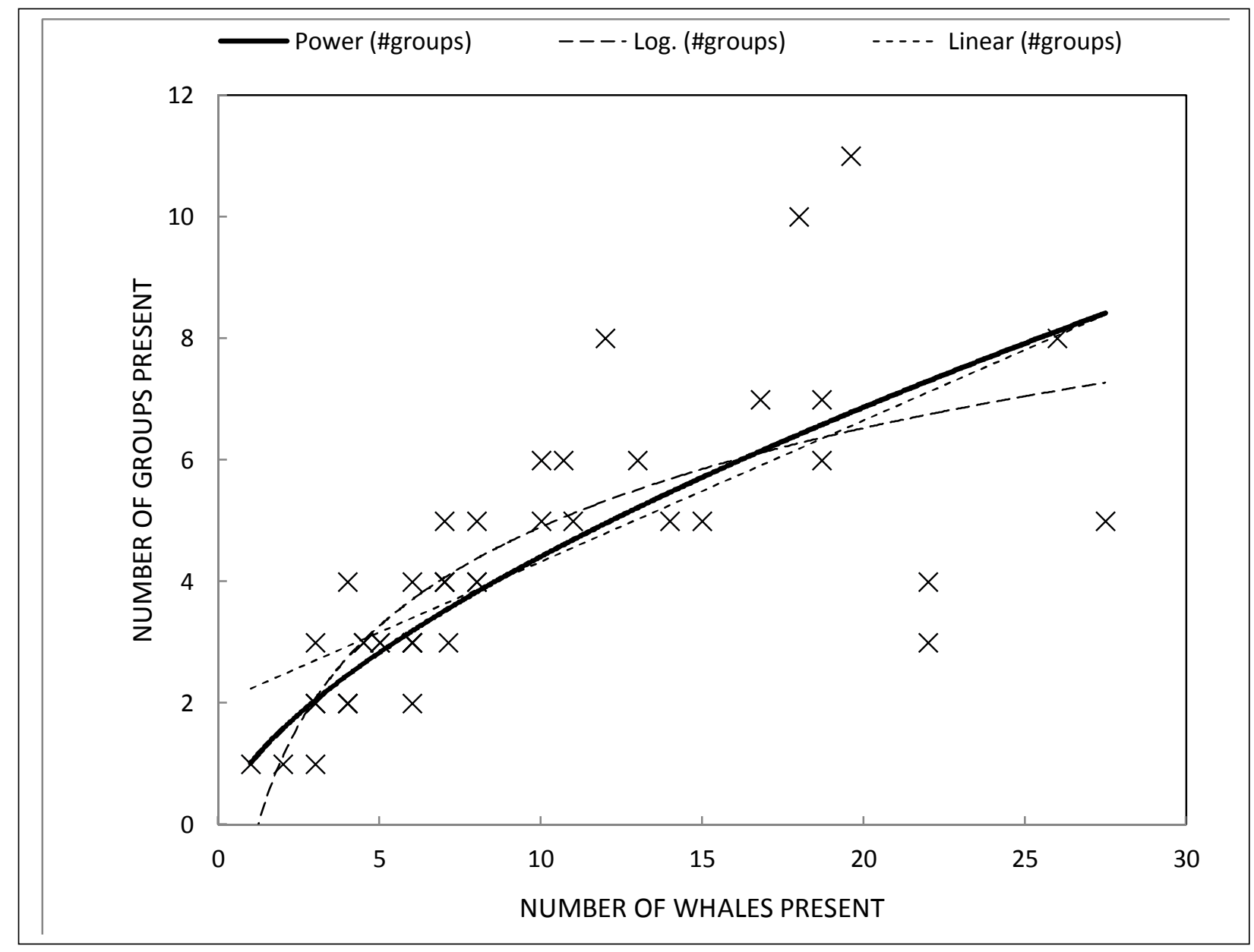

Figure 5. The relationship between number of whales present and numbers of groups present is best described by a power trendline $\left(R^{2}=0.6949\right)$, followed by a logarithmic trendline $\left(R^{2}=0.5001\right)$. A linear relationship $\left(R^{2}=0.4953\right)$ gives the worst fit. 


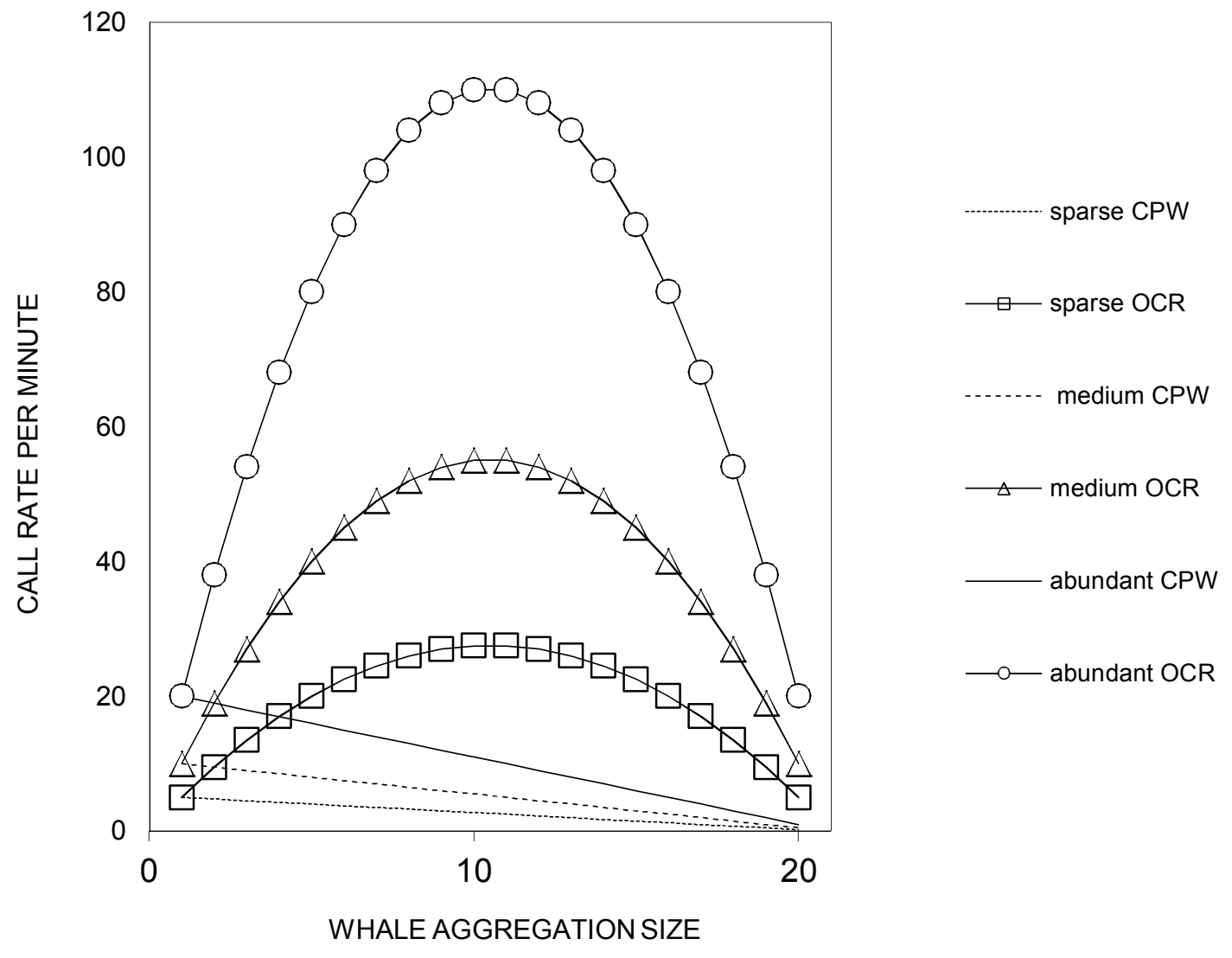

Figure 6. A simulated model of call rate per whale (CPW) and overall call rate for all whales present $(\mathrm{OCR})$, for increasingly large whale groups, demonstrates the logical outcome of an inverse relationship between CPW and whale aggregation size: this inevitably leads to an overall quadratic effect in the OCR. Hypothetical sparse (close dashes), medium (larger dashes) and abundant (solid line) call rates per whale (CPW), and sparse (squares), medium (triangles) and abundant (circles) overall call rates (OCR) are modeled. 


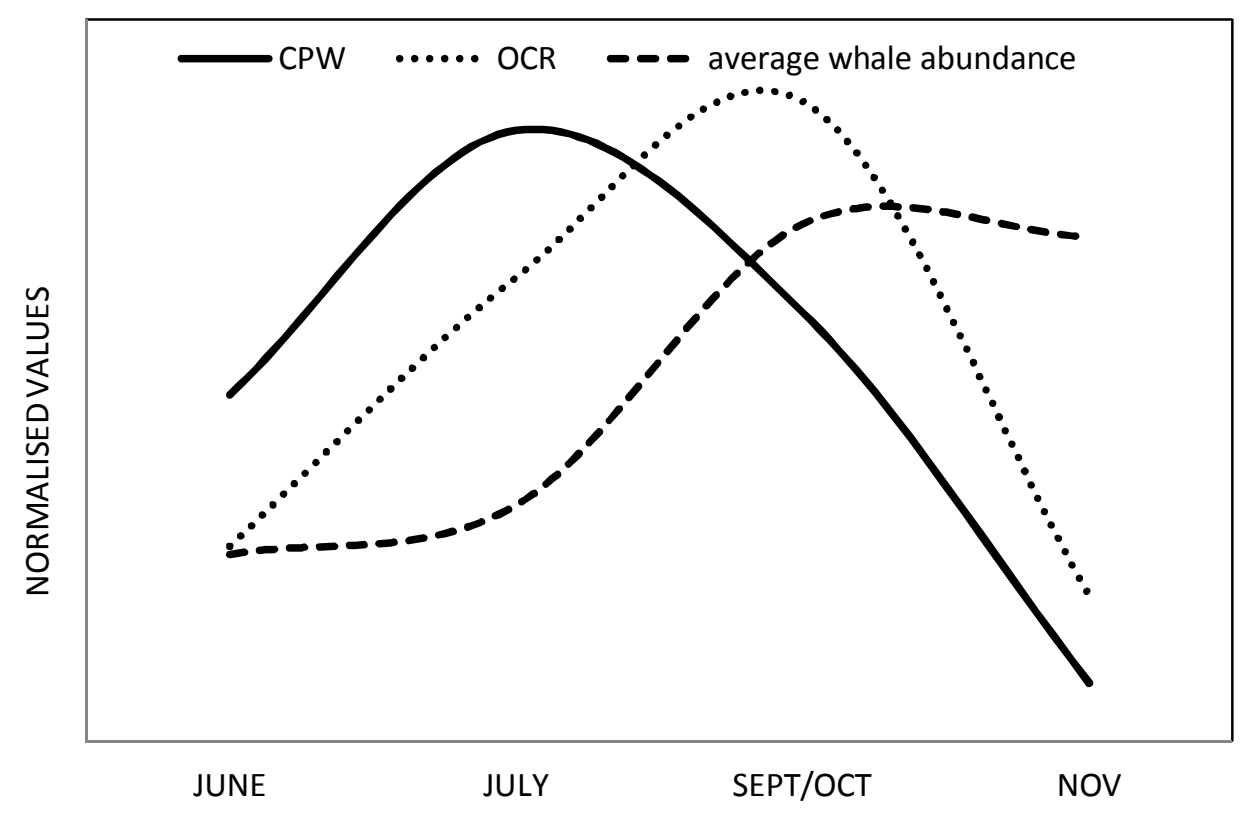

Figure 7. Overall call rate (OCR), call rate per whale (CPW), and whale abundance, for southern right whales in Walker Bay, South Africa, June through November. Observed data are normalised for comparison of trends. CPW clearly peaks in July, with low whale abundance, and is overtaken by OCR in September/October; both fall to their lowest levels with high whale abundance in November. 